\title{
Monte Carlo Ray Tracing based Method for Investigation of Multiple Reflection among Trees
}

\author{
Kohei Arai \\ Faculty of Science and Engineering \\ Saga University, Saga City, Japan
}

\begin{abstract}
Monte Carlo Ray Tracing (MCRT) method for investigation of the multiple reflection among trees is proposed. For the forest research (Leaf Area Index: LAI, Normalized Difference Vegetation Index: NDVI, forest type, tree age, etc.) with spaceborne based optical sensor data, some errors due to influences of the multiple reflection among trees on the estimation of at sensor radiance have to be considered. The influence is difficult to formulate in a radiative transfer equation. The proposed method allows estimating the influence. Through experiment with miniature sized forest, it is found that the proposed method is validated. It is also found that a few to more than $10 \%$ of influence due to multiple reflections among trees are anticipated. Furthermore, the influence on the estimation of at sensor radiance is clarified. The potentialities of the code are then depicted over different types of forests including coniferous and broadleaf canopies.
\end{abstract}

Keywords-Radiative transfer equation; Monte Carlo ray tracing: MCRT; multi reflection among trees; forest research; canopy reflectance; ellipse and cone shaped trees model

\section{INTRODUCTION}

Research on forest vegetation using earth observation satellite data is related to identification and identification of forest fires, logging areas surveys, forest maps, tree species discrimination, forest facies division map creation, etc. [1], carbon dioxide concentration, water vapor, latent heat in the ecosystem-atmosphere system There is a quantification of equal exchange and a tree structure analysis [2]. In addition, vegetation indexes, green ratio, amount of biomass, etc. are known as vegetation parameters that can be estimated from earth observation satellite data.

In addition to these, LAI (Leaf Area Index) for estimating growth and maintenance respiration of vegetation, leaf angle distribution characteristics, lignin (tree lignin, soil lignin cellulose index) for consumption and decomposition respiration calculation, growth, light utilization efficiency for photosynthetic activity (xanthophyll cycle, fluorescence), water stress (transpiration, leaf water content, etc.), temperature stress (leaf surface temperature), etc. for grasping maintenance process: LUE (Light Use Efficiency), photosynthesis effective Radiation: PAR (Photosynthetically Active Radiation), APAR (Absorbed PAR), fAPAR (Fraction of APAR), and basic production (NPP) have been estimated [3].

Lignin is synthesized by subjecting carbon compounds assimilated by photosynthesis (primary metabolism) to further metabolism (secondary metabolism). When a plant accumulated excessive reducing power in chloroplasts under conditions such as strong light, xanthophylls, which are auxiliary pigments of light-harvesting chlorophyll proteins, changed to substances with low light-harvesting efficiency, while becoming weak light. At that time, it changes to a substance with high light collection efficiency.

The purpose of this study is to clarify the optical characteristics of vegetation by using Monte Carlo method based on a three-dimensional tree structure model. From this characteristic, vegetation parameters such as tree shape and tree distance can be estimated. At this time, it is necessary to consider multiple reflection processes between trees and between trees and undergrowth, and absorption and multiple scattering processes by atmospheric air molecules and aerosols.

A model considering these has already been proposed by Goel \& Thompson [9], but only individual factors such as the effect of multiple reflection between trees and the effect of multiple reflection including trees and undergrowth have not been grasped. The relationship between the parameters is not always clear. Originally, the Monte Carlo method stochastically inserts photons into the atmospheric cell and simulates its optical path, so that it is possible to understand the influence of each factor.

In particular, this paper separately evaluates the effects of multiple reflections between trees and between trees and undergrowth. This reveals the error in estimating the tree reflectance and the tree distance, ignoring these effects. That is, the reflectance of the tree obtained from the Earth observation satellite image reflects these influences, and the reflectance that is truly desired can be obtained by considering these influences.

The same applies to tree shapes and tree distances estimated based on these. In order to evaluate the validity of the Monte Carlo method used in this study, we compared and compared it with the experimental results using a scale model of a forest in which two trees of two relatively small sizes with different tree shapes were arranged two-dimensionally. As a result, the validity of the Monte Carlo method was shown, and the effects of inter-tree multiple reflection and tree shape on the radiance at the upper edge of the atmosphere were clarified. In addition, the proposed method was applied to ASTER / VNIR data, and we were able to understand these effects, so the author reports here.

In the following chapter, related research works and theoretical background including motivation of the research are described together with research background. Section 3 details the Monte Carlo method. In particular, the geometrical and 
optical models of trees and undergrowth hypothesized on the sun and sensors and the ground surface are detailed. In addition, the types of optical path patterns are enumerated, and the optical path patterns of multiple reflections including tree shapes, trees, and trees and undergrowth that are of interest in this study are described in detail. Section 4 introduces the scale model experiment conducted to evaluate the validity of the Monte Carlo method. Furthermore, an application example to ASTER / VNIR data is shown. In Section 5, the author considers the influence of tree reflection and multiple reflections including trees and undergrowth and tree shape on tree distance estimation.

Then, the experimental method together with experimental results is described. After that concluding remarks and some discussions are also described.

\section{RELATED RESEARCH WORKS}

In relation to the forest investigation from space, studies have been conducted on the estimation of tree species, tree shape, distance between trees, vegetation density, etc. [4]. In most of these studies, based on a single horizontally homogeneous tree model layer, vegetation parameters in the layer are estimated [5].

In recent years, a three-dimensional vegetation structure model consisting of several layers has been proposed and has come to be considered when estimating canopy clamps, albedo, LAI, etc. that consider bidirectional reflectance characteristics [6]. In addition, a model that considers inhomogeneity in the horizontal and vertical directions has been proposed, and the three-dimensional vegetation structure, which is indispensable for studies such as radiation balance on the surface of vegetation, carbon dioxide exchange with optical absorption and other physical processes, is considered. The author started to do it [7].

On the other hand, problem solving methods for radiative transfer equation, there are the following research works,

A radiative transfer model based on three-dimensional vegetation structure has been proposed [8]. A radiative transfer model based on the Monte Carlo method has also been proposed, which is useful for improving the accuracy of analysis of vegetation and biological / physical parameters considering the three-dimensional vegetation structure [9]. Furthermore, studies such as estimating the amount of chlorophyll from the hyperspectral data using the inverse problem solution based on the leaf scale model of conifers have been conducted [10]. Adjacency effect of layered clouds estimated with Monte-Carlo simulation is investigated [11]. Non-linear mixture model of mixed pixels in remote sensing satellite images based on Monte Carlo simulation is proposed [12].

Forest parameter estimation by means of Monte Carlo simulations with experimental considerations (Estimation of multiple reflections among trees depending on forest parameters) is conducted [13] together with forest parameter estimation, by means of Monte-Carlo simulations with experimental consideration of estimation of multiple reflections among trees depending on forest parameters [14].
Micro traffic simulation with unpredictable disturbance based on Monte Carlo simulation is conducted for validation of effectiveness of the agent cars of Sidoarjo hot mudflow disaster is reported [15] together with Monte Carlo ray tracing simulation for bi-directional reflectance distribution function and grow index of tealeaves estimation [16].

Monte Carlo simulation of polarized atmospheric irradiance for determination of refractive index of aerosols is conducted [17]. Also, Monte Carlo ray tracing simulation of polarization characteristics of sea water which contains spherical and nonspherical shapes of suspended solid and phytoplankton is conducted [18].

Monte Carlo based non-linear mixture model of earth observation satellite imagery pixel data is proposed [19]. On the other hand, Monte Carlo ray tracing based sensitivity analysis of the atmospheric and oceanic parameters on the top of the atmosphere radiance is conducted [20].

Monte Carlo ray tracing based nonlinear mixture model of mixed pixels in Earth observation satellite imagery data is also proposed [21]. Comparison between linear and nonlinear models of mixed pixels in remote sensing satellite images based on Cierniewski surface Bi-Directional Reflectance Distribution Function: BRDF model by means of Monte Carlo ray tracing simulation is conducted [22].

Monte Carlo Ray Tracing: MCRT based knowledge base system for texture mapping together with height estimation using objects' shadow with high spatial resolution remote sensing satellite imagery data is proposed [23]. Meanwhile, Monte Carlo ray tracing based adjacency effect and nonlinear mixture model for remote sensing satellite image data analysis is conducted [24].

Method for aureole estimation refinement through comparisons between observed aureole and estimated aureole based on Monte Carlo Ray Tracing is proposed and validated with experiments [25].

\section{TheORETICAL BACKGROUND AND THE PROPOSED METHOD}

\section{A. Monte Carlo Method}

The Monte Carlo method is a method that treats sunlight as photons and probabilistically elucidates the behavior of photons in the atmosphere and on the ground surface [26]. Prerequisites are solar zenith angle, azimuth angle, sensor zenith angle, azimuth angle, observation wavelength, atmospheric cell size, atmospheric air molecule and aerosol optical thickness, surface reflectance: undergrowth and soil mixing (Lambertian surface) We give the tree size and the reflectance between trees (Lambertian surface).

Atmospheric composition molecules were only air molecules and aerosols. Since the observation wavelength range was set to visible near infrared, we decided to deal only with the composition that mainly contributes to absorption and scattering in this wavelength range. The ground surface is composed of trees and undergrowth. Trees have tree shapes such as spheres, ellipsoidal spheres, cylinders, and cones [27], [28]. 
In this study, from the viewpoint of the ease of constructing a scale model of a forest for the validation of the Monte Carlo method, the tree shapes that were relatively easily available were limited to elliptic spheres and cones. The photon is input to the atmospheric cell according to the following algorithm, the photon is counted when it is absorbed by the ground surface, trees, the atmosphere, and when it is captured in the sensor instantaneous visual field, and the optical path of the photon is terminated.

The photon is input to the atmospheric cell according to the following algorithm, the photon is counted when it is absorbed by the ground surface, trees, the atmosphere, and when it is captured in the sensor instantaneous visual field, and the optical path of the photon is terminated.

1) Assume the three-dimensional calculation cell shown in Fig. 1.

2) Photons are randomly generated and are incident from the top of the cell according to the solar zenith angle and azimuth angle.

3) Photons travel according to a free path determined by the optical thickness of the atmosphere.

4) If the end point of the free process of photons is in the atmosphere, either one of the photon molecules and the aerosol will be at a probability with the probability depending on the optical thickness of the aerosol.

5) Calculate the scattering angle of the photon and the remaining optical path length according to the scattering characteristics (phase function) of the air molecule and aerosol.

6) If the free process end point of the photon passes through the ground surface (trees / undergrowth), find the intersection with the tree / undergrowth, and reflect / absorb it. The reflection component reflects the remaining optical path length of the free process up to the intersection from the intersection.

7) If the end point of the free path of the photon is outside the cell, the photon is moved to the rest of the free path from the surface located exactly opposite the intersection of the free path vector and the cell wall surface.

8) The radiance at the top of the atmosphere is obtained based on the number of photons emitted from the upper surface of the cell into the instantaneous field of view of the sensor. The photon exiting the instantaneous visual field ends the optical path of the photon at that point.

9) A free process of photons and a log for each optical path are left so that the optical path locus of the photon can be traced.

For the random numbers, we use Mersenne Twister [29], which has a period of $2^{19937}-1$ and is evenly distributed in a 623-dimensional hypercube. The number of photons and the number of random numbers generated are determined by numerical experiments. The radiance at the top of the atmosphere is stable when the number of photons exceeds 100,000 , so the number of photons is set to 100,000 .

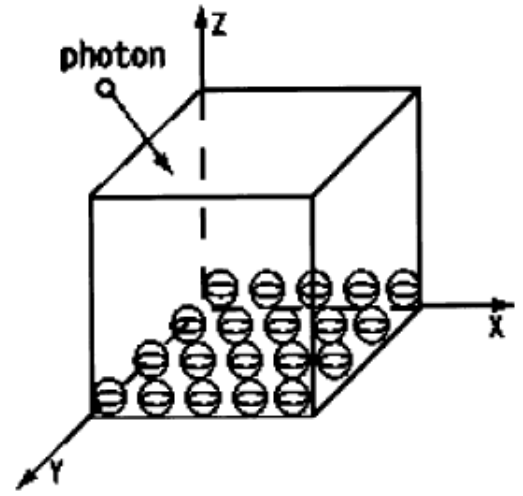

Fig. 1. Calculation Cell of Monte Carlo Simulation (Ellipsoidal Surface Objects are Trees).

Fig. 2 shows the possible optical paths of photons. The scattered light that is scattered only by the atmosphere and does not reach the ground surface is shown in Fig. 2(a), and the direct light that directly reaches the forest vegetation on the ground surface from the sun is shown in Fig. 2(b). Light path reaching the ground surface after scattering by the atmosphere Fig. 2(c) and Fig. 2(d) show the light path reaching the ground surface after multiple scattering by the atmosphere and undergoing multiple reflections between trees.

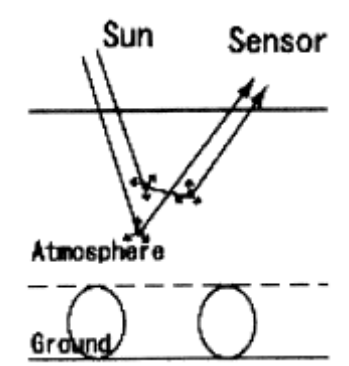

(a) Path Radiance.

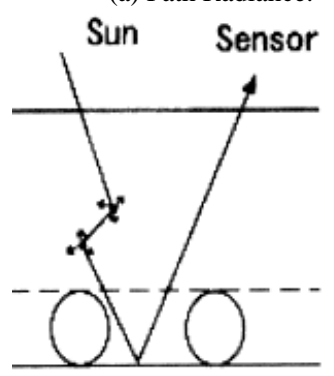

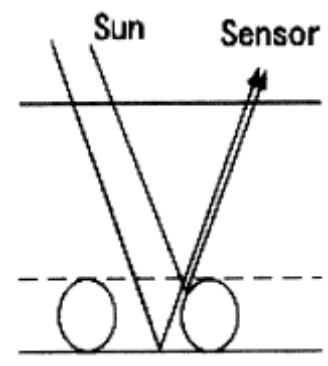

(b) Reflected Directly.

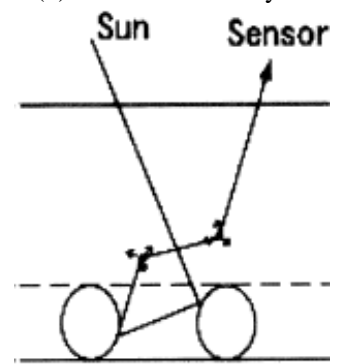

(c) Reflected by Sky Light and Ground. (d)Multiple Reflection among Trees.

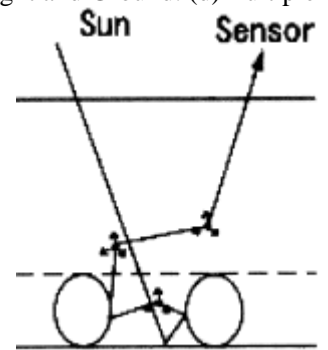

(e) Multiple Reflection among Trees, Sky Light and Ground.

Fig. 2. The Sun-atmosphere-ground Paths (Each Tree is Treated as each Individual Ellipse: Deciduous or cone: Coniferous Trees). 
Fig. 2(e) shows the optical paths of trees and undergrowth, or multiple reflections between trees and multiple scattering due to the atmosphere after reaching the ground surface. These optical path types can be grasped for their contributions by performing atmospheric top radiance calculation for each optical path in Monte Carlo simulation.

\section{B. Input Parameter}

Photons are transmitted and scattered depending on the preset optical thickness of the air molecules and aerosol. In the case of scattering of air molecules, it is scattered according to the scattering phase function based on Rayleigh scattering, and in the case of aerosol, it is scattered according to the phase function based on Mie scattering.

The multiple refractive indices of the aerosol were 1.44 for the real part and 0.005 for the imaginary part. These are the values obtained by actually measuring in the winter (December 13, 2003) around Saga University using PROM-III (Japan) Sky Radiometer POM-III. The particle size distribution was the Junge distribution and the Junge parameter was 3 . These are also the actual measured values around Saga University on the same day.

The aerosol scattering phase function was obtained using MODTRAN 4.0 Mie code (Software code to obtain scattering phase function by Mie scattering). Fig. 3 and 4 show the measured values of the real and imaginary parts of the complex refractive index and the Junge parameter, and the scattering phase function. The atmospheric optical thickness used in the Monte Carlo method is the measured value. The measured values are shown in Fig. 5. The surface of the tree is a Lambertian surface with uniform reflectance, and its shape is considered as an elliptical sphere or a cone as shown in Fig. 6.

Considering the distance between trees, we assumed a forest in which these trees were arranged in a grid. The state of the undergrowth was a Lambertian surface with a uniform reflectance. It is possible to consider bidirectional reflection distribution function (BRDF), Mineralt reflection law, trees based on fractal shape on the surface of trees and undergrowth [30], [31], where Lambert is used as the first approximation. The author assumed a face. In addition to these, measured values were used for the reflectance and shape of trees used in the Monte Carlo method.

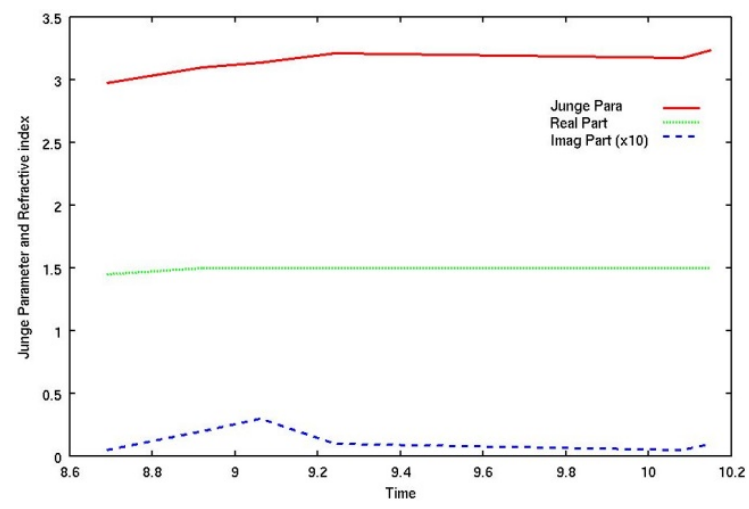

Fig. 3. Estimated Junge Parameter of Size Distribution and Real and Imaginary Part of Refractive Index of Aerosol with the Measured Data using POM-III of Sky-radiometer at the Saga University and its Surrounding on Dec. 132003.

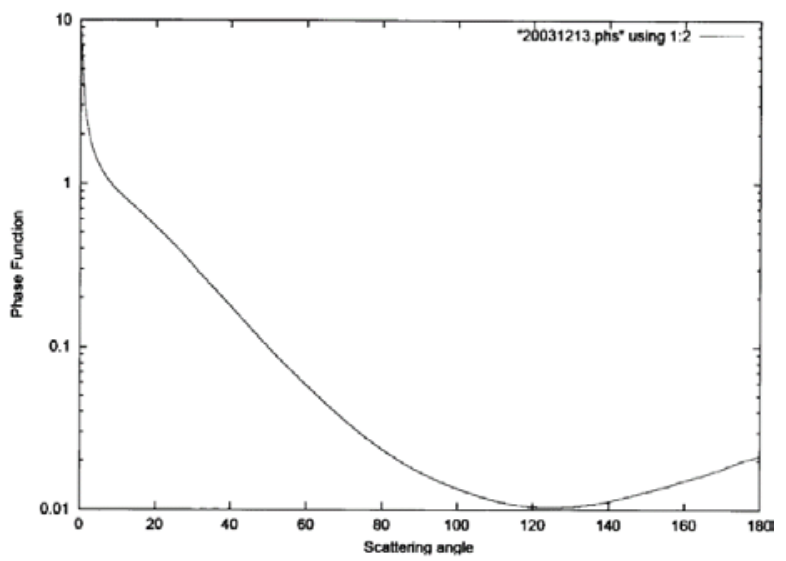

Fig. 4. Aerosol Phase Function used for Monte Carlo Simulation.

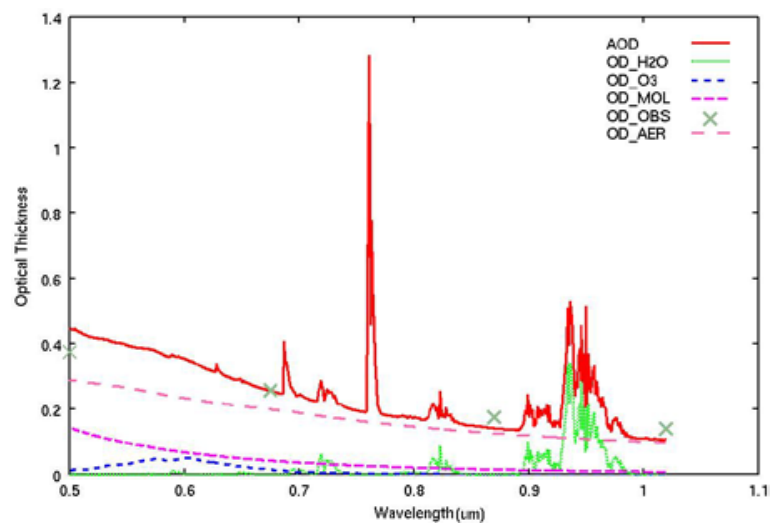

Fig. 5. Measured Optical Depths of Total Atmosphere (AOD), Water Vapor (OD_H20), Column Ozone (OD_03), Molecule (OD_MOL), and Aerosol (OD_AER) as well as Observed Optical Depth (OD_OBS) at Saga Test Site in Japan on Dec. 152004.

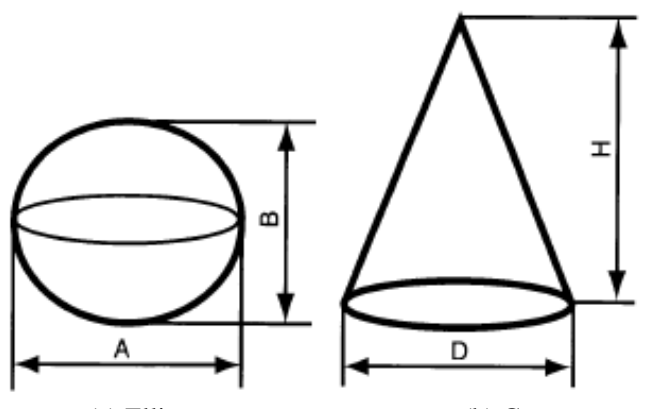

(a) Ellipse

(b) Cone

Fig. 6. Two Kinds of Tree Models.

The author considers the parameter setting by using a comprehensive e-learning system with simulation capabilities for understanding of complex equations [32].

\section{Output Parameters}

The output parameters in the Monte Carlo method are the atmospheric top radiance and 10 types of photon statistical data:

1) The total number of photons incident on the cell from the cell top surface.

2) The number of photons emitted from the cell top surface, and trees. 
3) The number of photons reflected by trees.

4) The number of photons reflected by undergrowth.

5) The number of photons absorbed by undergrowth.

6) The number of photons scattered by aerosols.

7) The number of photons absorbed by aerosol.

8) The number of photons scattered by air molecules.

9) The number of photons absorbed by air molecules.

\section{EXPERIMENT}

\section{A. Method for Experiment}

Since it is difficult to measure multiple reflections between trees and between trees and undergrowth in natural forests, we decided to use the Monte Carlo method. For that purpose, the validity of the Monte Carlo method must be proved. Two types of relatively small size tree-shaped trees simulating a forest were arranged two-dimensionally, and a lawn was spread as undergrowth, and a black cloth was spread around it. The experimental configuration is shown in Fig. 7(a).

The experiment site is the center of the Saga University Athletic Ground, and the ground is surrounded by trees with a height of about $7 \mathrm{~m}$, but the skyline is within about 2 degrees elevation when viewed from the center. The spectroradiometer used for measuring the ground surface reflectance was MS-720 manufactured by Eiko Instruments Co., Ltd. This is to measure the radiance by separating the wavelength range from 350 to $1050 \mathrm{~nm}$ into 256 channels at $3.3 \mathrm{~nm}$ wavelength intervals. As a standard white plate, Spectralon manufactured by Labsphere, USA was used. To monitor the direct light of the sun, GER2600 (measured radiance in the wavelength range of 400 to $2600 \mathrm{~nm}$ at $2 \mathrm{~nm}$ intervals) manufactured by GER, USA was used.

The height of the tree height of the MS720 radiometer with an opening angle of 45 degrees was set to $1.8 \mathrm{~m}$. Therefore, the measurement range is within a circle with a radius of $74.56 \mathrm{~cm}$ centered directly under the radiometer. The lawn is a square with a side of $1.8 \mathrm{~m}$, and the black cloth is a square with a side of $2.5 \mathrm{~m}$. The above two types of trees were placed on this lawn. An overview of the tree is shown in Fig. 7(b).

Tree No. 1 is a conifer (Jerichoides, lateral diameter: $13 \mathrm{~cm}$, vertical diameter: $12 \mathrm{~cm}$ ), and No. 2 is a conifer (gold crest, bottom diameter: $11 \mathrm{~cm}$, height: $26 \mathrm{~cm}$ ). These were arranged two-dimensionally as shown in Fig. 8 to construct a forest scale model. In addition, Sky Radiometer POM-III is installed on the rooftop of the Faculty of Science and Engineering Building No. 7 (4 floors) about $100 \mathrm{~m}$ away from the ground, and direct observation of the sun, scattering, and ambient light is performed every day.

\section{B. Experimental Results}

Fig. 9 shows the spectral reflectance characteristics when the distance between trees is changed from $8 \mathrm{~cm}$ to $30 \mathrm{~cm}$ in $2.5 \mathrm{~cm}$ increments. The parameter in the legend in the figure is the distance between trees in $\mathrm{cm}$. This is the characteristic of No. 1 tree, but it is almost the same for No. 2. This is a typical spectral reflectance characteristic of vegetation in which the reflectance is high in the green wavelength, low in the red wavelength, and highest in the near infrared wavelength range.

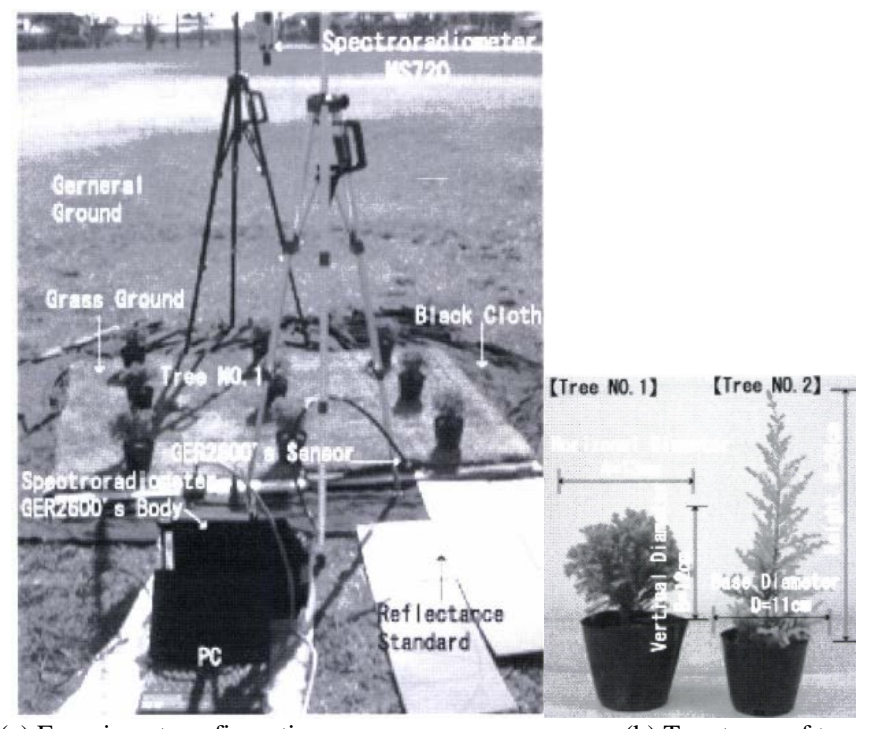

(a) Experiment configuration

(b) Two types of trees

Fig. 7. Outlook of the Forested Trees and Grasses on the Land Surface together with the Measuring Instruments for Surface Reflectance (Spectralon : Standard Plaque, MS-720 and GER260O : Spectroradiometers).

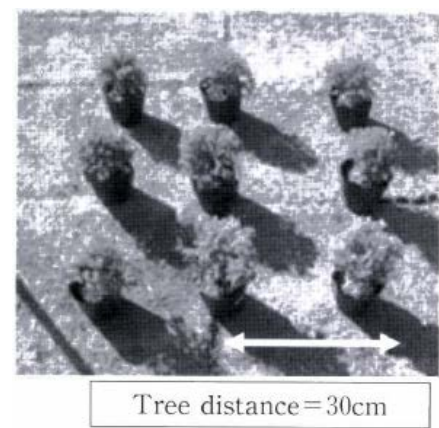

(a) Tree No.1

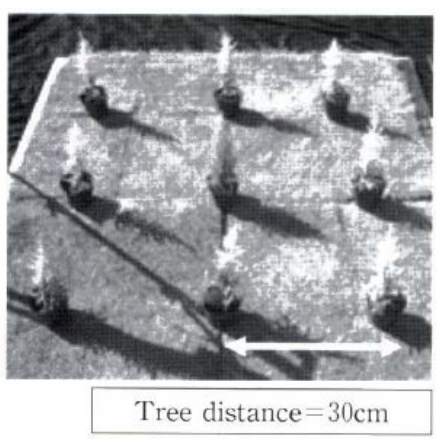

(b) Tree No.2
Fig. 8. Trees used in Experiment.

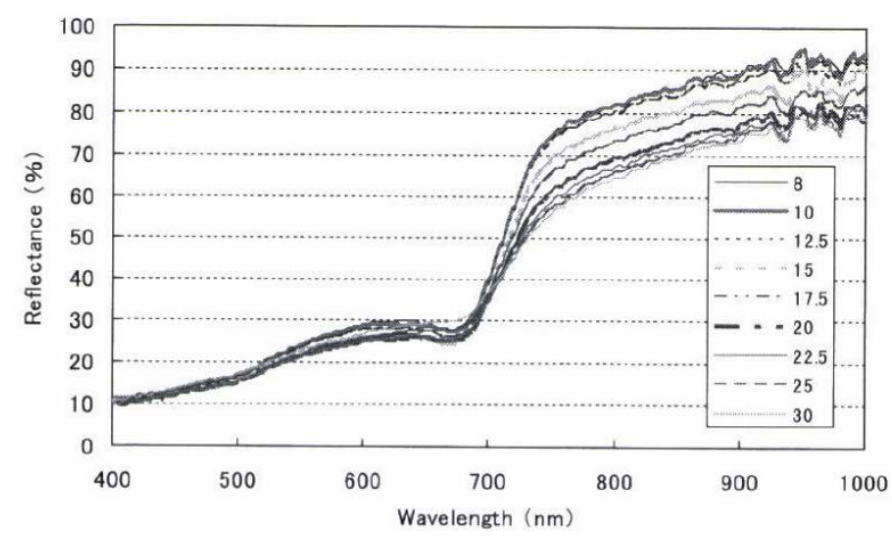

Fig. 9. Measured Reflectance of the Scale Model of the Two Dimensionally Aligned Trees (No.1) with the Tree Distance Ranges from 8 to 30cm (Legend shows the Tree Distance).

For the purpose of this study to clarify the effect of multiple reflections between trees and undergrowth on the upper radiance of the atmosphere, the reflectance changes significantly with the distance between trees 750,800,900,1000 $\mathrm{nm}$ (wavelengths that are less absorbed by atmospheric air 
molecules). Attention was paid to the reflectance at. Similarly, the spectral reflectance of grass, which is assumed to be undergrowth, was also measured.

Atmospheric optical thickness and geometric relationships with the sun, ground surface and sensors were investigated. Simultaneously with this experiment, we measured the optical thickness of the atmosphere at wavelengths of 368,500,675,778,862 nm, which avoids absorption of air molecules. The measuring instrument is MICROTOPS-II (Langley plot specification) manufactured by Solar Spectrum.

Fig. 5 shows the measurement results of the optical thickness. From this, the optical thickness of the aerosol was estimated by subtracting the total amount of ozone measured by scattering of air molecules (Rayleigh scattering) as a function of atmospheric pressure and wavelength and MICROTOPS-II (specification of ozone and water vapor) and absorption by total amount of water vapor.

The geometric parameters of the sun, ground surface and sensor were also obtained. These parameters are shown in Tables I and II. Here, the reflectance of a tree is measured from the top of the tree, and it is assumed that this reflectance is the same for all surfaces of a cone and an ellipsoid (Lambertian surface). Strictly speaking, there are differences in reflectance between the bottom and side surfaces of the cone, the upper and lower hemispheres of the ellipsoid, and the effects of multiple reflections, transmission, and scattering inside this shape, but the assumption was adopted as the first approximation.

Experiments using a forest scale model were compared with simulation results using the Monte Carlo method. Fig. 10(a) and 10(b) show a comparison of the distance between trees and sensor radiance characteristics by experiments using a forest scale model and simulations based on the Monte Carlo method. In the figure, the measured data (triangle, square, and diamond mark) and the data calculated by Monte Carlo simulation (solid line and broken line) are shown.

The top characteristic is the sensor radiance at $1000 \mathrm{~nm}$, followed by 900,800 and $750 \mathrm{~nm}$. It can be seen that the sensor radiance decreases monotonically as the tree distance increases. This is probably because the effect of multiple reflections between trees and between trees and undergrowth on the sensor radiance decreases with the distance between trees. Moreover, the Monte Carlo method is valid because the two agree very well.

The parameters used for the Monte Carlo method were adapted to those of the forest scale model. That is, the cell size was a cube with the height of the light receiving part of the spectrometer as one side, the sensor aperture was 45 degrees full angle, and the other parameters shown in Tables I and II were used.

As a result, in Tree No. 1, the percentage of the total number of photons that reached the upper edge of the atmosphere after two or more reflections between trees at 750 $\mathrm{nm}$ was $8 \%$, and reached $10 \%$ at a wavelength of $1000 \mathrm{~nm}$. I found out that similarly, in the case of Tree No. 2, it was found to be $2.2 \%$ at $750 \mathrm{~nm}$ and $3.1 \%$ at $1000 \mathrm{~nm}$. In other words, $10 \%$ of the reflectance in the near infrared of a forest on an ellipsoidal sphere based on satellite visible and near infrared radiometer data is due to the multiple reflections between trees, and the actual reflectance is about $10 \%$ lower. It can be said to be a value.

TABLE I. INPUT PARAMETERS FOR TREE NO. 1

\begin{tabular}{|c|c|c|c|c|}
\hline Parameter & 750nm & $800 \mathrm{~nm}$ & $900 \mathrm{~nm}$ & $1000 \mathrm{~nm}$ \\
\hline \multicolumn{5}{|l|}{ Reflectance } \\
\hline Grass reflectance & $29 \%$ & $33 \%$ & $42 \%$ & $53 \%$ \\
\hline Tree reflectance & $75 \%$ & $82 \%$ & $89 \%$ & $93 \%$ \\
\hline \multicolumn{5}{|l|}{ Optical depth } \\
\hline Molecule & 0.045 & 0.04 & 0.04 & 0.03 \\
\hline Aerosol & 0.045 & 0.04 & 0.04 & 0.03 \\
\hline \multicolumn{2}{|c|}{ Sun-Sensor Geometry } & \multicolumn{2}{|l|}{ Canopy properties } & \\
\hline Solar zenith angle & $41^{\circ}$ & $\begin{array}{l}\text { Horizontal diameter } \\
\text { (A) }\end{array}$ & $13 \mathrm{~cm}$ & \\
\hline $\begin{array}{l}\text { Solar azimuth } \\
\text { angle }\end{array}$ & $15^{\circ}$ & $\begin{array}{l}\text { Vertical diameter } \\
\text { (B) }\end{array}$ & $12 \mathrm{~cm}$ & \\
\hline View zenith angle & $0^{\circ}$ & & & \\
\hline
\end{tabular}

TABLE II. INPUT PARAMETERS FOR TREE NO. 2

\begin{tabular}{|c|c|c|c|c|}
\hline Parameter & $750 \mathrm{~nm}$ & $800 \mathrm{~nm}$ & $900 \mathrm{~nm}$ & $1000 \mathrm{~nm}$ \\
\hline \multicolumn{5}{|l|}{ Reflectance } \\
\hline Grass reflectance & $29 \%$ & $33 \%$ & $42 \%$ & $53 \%$ \\
\hline Tree reflectance & $65 \%$ & $75 \%$ & $84 \%$ & $92 \%$ \\
\hline \multicolumn{5}{|l|}{ Optical depth } \\
\hline Molecule & 0.045 & 0.04 & 0.04 & 0.03 \\
\hline Aerosol & 0.045 & 0.04 & 0.04 & 0.03 \\
\hline \multicolumn{2}{|l|}{ Sun-Sensor Geometry } & \multicolumn{2}{|l|}{ Canopy properties } & \\
\hline Solar zenith angle & $43^{\circ}$ & Base diameter (C) & $11 \mathrm{~cm}$ & \\
\hline Solar azimuth angle & $29^{\circ}$ & Height (D) & $25 \mathrm{~cm}$ & \\
\hline View zenith angle & $0^{\circ}$ & & & \\
\hline
\end{tabular}

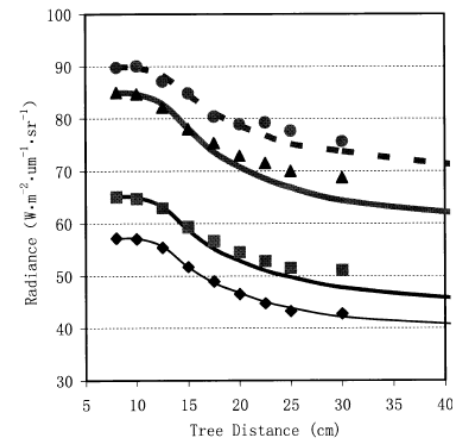

(a)

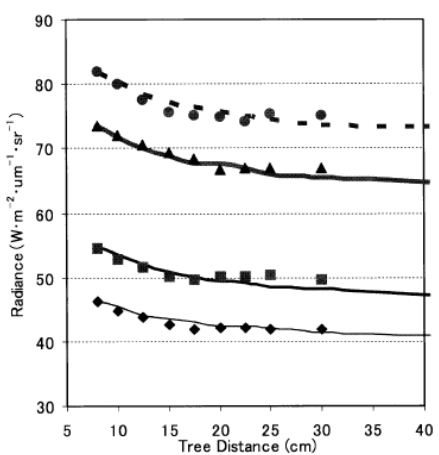

(b)
Fig. 10. At-Sensor Radiance from the Scale Model of Trees and Grasses for Tree No.1 ((a): Left) and that for Tree No.2; ((b): Right). Upper Curve shows Radiance at $1000 \mathrm{~nm}$ Followed by 900, 800, and 750nm. Solid and Dotted Lines show the Calculated Radiance based on Monte Carlo Simulation While Diamond, Square and Triangles show the Experimental Radiance with the Scale Model. 
It was also found that this effect was less in the case of conical forests, which was a few percent. This effect depends on the distance between trees, and it can be seen that the multiple reflections between trees decrease rapidly as the distance between trees increases. From the effect of these multiple reflections between trees when evaluated by the ratio with the radiance at the upper edge of the total atmosphere, the effect of multiple reflections between trees can be inferred to be from several\% to $10 \%$. The effect of this multiple reflection between trees depends on the tree shape, distance between trees, tree species, etc. In this experiment, we examined only conifers, but in the case of broadleaf trees with a larger LAI, It can be inferred that the influence is even greater in the case of mixed forests when they are closer.

\section{CONCLUSION}

A radiation model of the atmosphere and forest vegetation was constructed by the Monde Carlo method. This makes it possible to evaluate the effects on the upper atmospheric radiance of only multiple reflections between trees and between trees and undergrowth that were not considered in the existing model. The validity of the Mon-Carlo method used in this study was confirmed by comparison with experimental values by a forest scale model in the field. Experiments using a forest scale model were performed by changing the distance between trees and the tree shape.

Comparing this experimental value and the simulation result by the Monte Carlo method, it was found that the radiance tends to gradually decrease as the distance between trees increases. This is a tendency that changes in reflectance due to changes in area coverage of undergrowth and trees overlap with the effects of multiple reflections between trees. And the effect of multiple reflections including trees and undergrowth can be understood.

The effect of the optical path that contributes to this multiple reflection on the radiance at the top of the atmosphere can be grasped from the Monte Carlo method, and it was confirmed that it ranges from several\% to several tens of $\%$ (this effect also varies depending on the tree shape, It was found that it was about $10 \%$ in the case of an elliptical sphere and about $4 \%$ in the case of a cone).

This effect is in the near-infrared wavelength region and less in the visible wavelength region. In conclusion, the effect of multiple reflections between trees depends on tree shape, distance between trees, tree species (even single tree species change depending on age and season), LAI: Leaf Area Index, leaf angle distribution, etc. However, it is difficult to say how much it is, but it can be inferred that the impact is from several $\%$ to $10 \%$.

Therefore, it can be seen that there is a risk that the reflectance of trees obtained from satellite images is estimated to be several to several tens of percent higher than the actual reflectance. Applying this method to understand the effects of multiple reflections between trees and undergrowth of trees to ASTER / VNIR band 3 data, the difference between the satellite data and the simulation results on bare land (no effect of multiple reflections) was $1.19 \%$.
However, it is confirmed that it is as much as $7.47 \%$ in the coniferous forest (which is affected by multiple reflections). Therefore, most of the $7.47 \%$ difference is considered to be due to the multiple reflections between trees. It was also confirmed that the difference between the radiance obtained from the ASTER / VNIR band 3 data and that obtained from the Monte Carlo method was $11.28 \%$ when the tree shape was changed to an ellipsoidal shape. This fact suggests that tree shape discrimination is possible using these differences.

However, in the simulation by the Monte Carlo method, the tree shape, the distance between trees, etc. are provisionally set, and it is hard to say that the influence of the terrain inclination, undulation, etc. is strictly considered. Therefore, the effect of multiple reflections of several $\%$ to $10 \%$ is only an approximate number, but it is possible to argue that it is necessary to consider this effect when estimating reflectance of trees, distance between trees, and tree shape.

\section{FUtURE RESEARCH WORKS}

In the future, the author will conduct another experiment with actual remote sensing satellite data for the validation of the proposed method.

\section{ACKNOWLEDGMENT}

The author would like to thank Dr. Yaliu Ding of former student of Saga University for her contribution of this study. The author, also, would like to thank Professor Dr. Hiroshi Okumura and Professor Dr. Osamu Fukuda for their valuable discussions.

\section{REFERENCES}

[1] Hall, F.G., Knapp, D.E. and Huemmrich, K.F., Physically based classification and satellite mapping of biophysical characteristics in the southern boreal forest, Journal of Geophysical Research, 102, D24, 29567-29580, 1997.

[2] Baldcchi, D., Kelliher, F.M., Black, T.A. and Javis, P.G., Climate and vegetation controls on boreal zone energy exchange, Global Change Biology, 6, Suppl. 1, 69-83, 2000.

[3] Nobel, P.S. and Long, S.P., Canopy structure and light interception, In : J. Coombs, D.O. Hall, S.P. Long and J.M.O. Scurlock (Edt.), Techniques in bioproductivity and photosynthe sis (2nd edt.) Oxford : Pergamon, 1985.

[4] Chen, J.M. and Cihlar, J., Plant canopy gap size analysis theory for improving optical mea surements of leaf area index, Applied Optics, 34, 6211-6222, 1995.

[5] Walthall, C.L., Loechel, S.E., Huemmrich, K. F., Brown de Colstoum, E., Chen, J., Markham, 65 B.L., Miller, J., and Walter-Shea, E.A., Spectral information content of the boreal forest, I : G. Guyot, and T. Phulpin (Edt.) Proc. of Physical Measures and Signatures in Remote Sensing, 607-611, 1997.

[6] Running, SW., and Coughlan J.C., A general model of forest ecosystem processes for regional applications : 1 . Hydorologic balance, canopy gas exchange and primary production processes, Ecological Modeling, 42, 125-154, 1988.

[7] Chen, J.M., Liu, J., Leblanc, S.G., Lacaz, R. and Roujean, J.L., Multiangular optical remote sensing for assessing vegetation structure and carbon absorption, Remote Sensing of Environ ment, 84, 4, 516-525, 2003.

[8] Gastellu-Etchegorry, J.P., Guillevie, P., Zagolski, F., Demarez, V., Trichon, V., Deering, D., and Leroy, M., Modeling BRF and radiation regime of boreal and tropical forests : I. BRF. Remote Sensing of Environment, 68, 3, 281-316, 1999. 
[9] Goel, N.S. and Thompson, RI., A snapshot of canopy reflectance models and a universal model for the radiation regime, Remote Sensing Reviews, 18, 197-225, 2000.

[10] Zarco-Tejada, P.J. Miller, JR., Harron, J., Hu, B., Noland, TI, Goel, N.S., Mohammad, G. H. and Sampson, P., Needle chlorophyll content estimation through model inversion using byperspectral data from boreal conifer forest canopies, Remote Sensing of Environment, 89, 189-199, 2003.

[11] Kohei Arai, Adjacency effect of layered clouds estimated with MonteCarlo simulation, Advances in Space Research, Vol.29, No.19, 18071812, 2002.

[12] Kohei Arai, Non-linear mixture model of mixed pixels in remote sensing satellite images based on Monte Carlo simulation, Advances in Space Research, 41, 11, 1715-1723, 2008.

[13] Yaliu Ding and Kohei Arai Forest parameter estimation by means of Monte Carlo simulations with experimental considerations -Estimation of multiple reflections among trees depending o n forest parametersOnline publication from Advances in Space Research, Elsevier 2008.

[14] Yaliu Ding, Kohei Arai, Forest parameter estimation, by means of Monte-Carlo simulations with experimental consideration of estimation of multiple reflections among trees depending on forest parameters, Advances in Space Research, 43, 3, 438-447, 2009.

[15] Kohei Arai, Tri Harsono, Achmad Basuki, Micro traffic simulation with unpredictable disturbance based on Monte Carlo simulation and effectiveness of the proposed agent cars of Sidoarjo hot mudflow disaster, Journal of EMITTER, 1, 1, 10-19, 2010.

[16] Kohei Arai, Monte Carlo ray tracing simulation for bi-directional reflectance distribution function and grow index of tealeaves estimation, International Journal of Research and Reviews on Computer Science, 2, 6, 1313-1318, 2011.

[17] Kohei Arai, Monte Carlo simulation of polarized atmospheric irradiance for determination of refractive index of aerosols, International Journal of Research and Review on Computer Science, 3, 4, 1744-1748, 2012.

[18] Kohei Arai, Y.Terayama, Monte Carlo ray tracing simulation of polarization characteristics of sea water which contains spherical and non-spherical shapes of suspended solid and phytoplankton, International Journal of Advanced Computer Science and Applications, 3, 6, 85-89, 2012.

[19] Kohei Arai, Monte Carlo based non-linear mixture model of earth observation satellite imagery pixel data, International Journal of Advanced Computer Science and Applications, 3, 8, 18-22, 2012.

[20] Kohei Arai, Monte Carlo ray tracing based sensitivity analysis of the atmospheric and oceanic parameters on the top of the atmosphere radiance, International Journal of Advanced Computer Science and Applications, 3, 12, 7-13, 2012.

[21] Kohei Arai, Monte Carlo ray tracing based nonlinear mixture model of mixed pixels in Earth observation satellite imagery data, International Journal of Advanced Computer Science and Applications, 4, 1, 148-152, 2013.

[22] Kohei Arai, Comparison between linear and nonlinear models of mixed pixels in remote sensing satellite images based on Cierniewski surface BRDF model by means of Monte Carlo ray tracing simulation, International Journal of Advanced Research in Artificial Intelligence, 2, 4, 1-7, 2013.
[23] Kohei Arai, Monte Carlo Ray Tracing: MCRT based knowledge base system for texture mapping together with height estimation using objects' shadow with high spatial resolution remote sensing satellite imagery data, International Journal of Advanced Research in Artificial Intelligence, 2, 6, 51-55, 2013.

[24] Kohei Arai, Monte Carlo ray tracing based adjacency effect and nonlinear mixture model for remote sensing satellite image data analysis, International Journal of Advanced Research in Artificial Intelligence, 2, 6, 56-64, 2013.

[25] Kohei Arai, Method for aureole estimation refinement through comparisons between observed aureole and estimated aureole based on Monte Carlo Ray Tracing, International Journal of Advanced Research in Artificial Intelligence, 2, 12, 1-8, 2013.

[26] Kohei Arai: Self-study remote sensing, Morikita Publishing, 2001.

[27] Sadamoto Watanabe: Sociology of trees, The University of Tokyo Press, pp.74-78, 1994.

[28] Toshio Tsutsumi: Forest Ecology, Asakura Shoten, pp.37-42, 1989.

[29] Mersenne Twister (MT), http://www.mathsci.hiroshima-u.ac.j p/ m$\mathrm{mat} / \mathrm{MT} / \mathrm{mt} . \mathrm{html}$.

[30] Zarco-Tejada, P.J., Hyperspectral remote sensing of closed forest canopies : Estimation of chlorophyll fluorescence and pigment con tent. PhD Dissertation. Graduate Program in Earth and Space Science. York University, Toronto, 2000.

[31] Disney, M.I., P. Lewis, P.R. North, Monte Carlo ray tracing in optical canopy reflectance modeling, International Forum on BRDF Spe cial Issue, Remote Sensing Reviews, pp.1-50, 2000.

[32] Kohei Arai, Comprehensive e-learning system with simulation capabilities for understanding of complex equations, International Journal of Advanced Computer Science and Applications IJACSA, 10, 11, 330-335, 2019.

\section{AUTHOR’s PROFILE}

Kohei Arai, He received BS, MS and PhD degrees in 1972, 1974 and 1982, respectively. He was with The Institute for Industrial Science and Technology of the University of Tokyo from April 1974 to December 1978 also was with National Space Development Agency of Japan from January, 1979 to March, 1990. During from 1985 to 1987, he was with Canada Centre for Remote Sensing as a Post Doctoral Fellow of National Science and Engineering Research Council of Canada. He moved to Saga University as a Professor in Department of Information Science on April 1990. He was a councilor for the Aeronautics and Space related to the Technology Committee of the Ministry of Science and Technology during from 1998 to 2000 . He was a councilor of Saga University for 2002 and 2003. He also was an executive councilor for the Remote Sensing Society of Japan for 2003 to 2005. He is a Science Council of Japan Special Member since 2012. He is an Adjunct Professor of University of Arizona, USA since 1998. He also is Vice Chairman of the Science Commission "A" of ICSU/COSPAR since 2008 then he is now award committee member of ICSU/COSPAR. He wrote 55 books and published 620 journal papers as well as 450 conference papers. He received 66 of awards including ICSU/COSPAR Vikram Sarabhai Medal in 2016, and Science award of Ministry of Mister of Education of Japan in 2015. He is now Editor-in-Chief of IJACSA and IJISA. http://teagis.ip.is.sagau.ac.jp/index.html. 